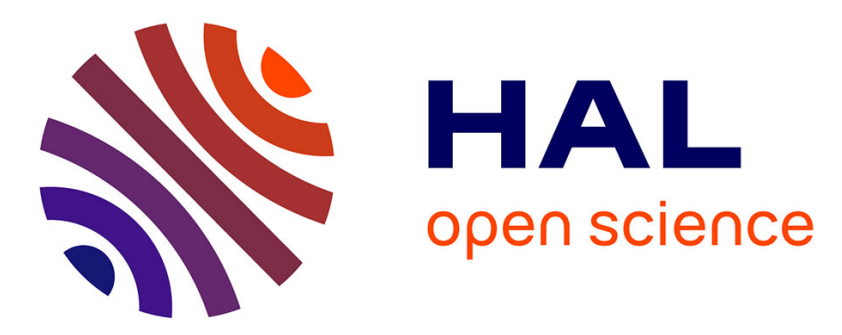

\title{
Approximate decomposition of some modulated-Poisson Voronoi tessellations
}

Bartlomiej Blaszczyszyn, René Schott

\section{To cite this version:}

Bartlomiej Blaszczyszyn, René Schott. Approximate decomposition of some modulated-Poisson Voronoi tessellations. [Research Report] RR-4585, INRIA. 2002. inria-00072000

\section{HAL Id: inria-00072000 https://hal.inria.fr/inria-00072000}

Submitted on 23 May 2006

HAL is a multi-disciplinary open access archive for the deposit and dissemination of scientific research documents, whether they are published or not. The documents may come from teaching and research institutions in France or abroad, or from public or private research centers.
L'archive ouverte pluridisciplinaire HAL, est destinée au dépôt et à la diffusion de documents scientifiques de niveau recherche, publiés ou non, émanant des établissements d'enseignement et de recherche français ou étrangers, des laboratoires publics ou privés. 


\section{N R I A}

INSTITUT NATIONAL DE RECHERCHE EN INFORMATIQUE ET EN AUTOMATIQUE

\section{Approximate decomposition of some modulated-Poisson Voronoi tessellations}

Bartłomiej Błaszczyszyn — René Schott

\section{$\mathbf{N}^{\circ} 4585$}

Octobre 2002

THÈME 1 



\title{
Approximate decomposition of some modulated-Poisson Voronoi tessellations
}

\author{
Bartłomiej Błaszczyszyn*, René Schott ${ }^{\dagger}$ \\ Thème 1 - Réseaux et systèmes \\ Projet TREC
}

Rapport de recherche $\mathrm{n}^{\circ} 4585$ - Octobre 2002 - 17 pages

\begin{abstract}
We consider the Voronoi tessellation of Euclidian space that is generated by an inhomogeneous Poisson point process whose intensity takes different constant values on sets of some finite partition of the space. Considering the Voronoi cells as marks associated to points of the point process, we prove that the intensity measure (mean measure) of the marked Poisson point process admits an approximate decomposition formula. The true value is approximated by a mixture of respective intensity measures for homogeneous models, while the explicit upper bound for the remaining term can be computed numerically for a large class of practical examples. By the Campbell formula, analogous approximate decomposition are deduced for the Palm distributions of individual cells. This approach makes possible the analysis of a wide class of non-homogeneous-Poisson Voronoi tessellations, by means of formulae and estimates already established for homogeneous cases. Our analysis applies also to the Poisson process modulated by an independent stationary random partition, in which case the error of the approximation of the double-stochastic-Poisson Voronoi tessellation depends on some integrated linear contact distribution functions of the boundaries of the partition elements.
\end{abstract}

Key-words: Voronoi tessellation, inhomogeneous Poisson point process, double stochastic Poisson point process, Boolean model, intensity measure, approximations, decomposability.

${ }^{*}$ ENS/INRIA and Mathematical Institute University of Wrocław, ENS, 45 rue d'Ulm, 75005 Paris, France. E-mail: Bartek.Blaszczyszyn@ens.fr

$\dagger$ LORIA and IECN, Université Henri Poincaré-Nancy 1, BP 239, 54506 Vandoeuvre-lès-Nancy, France. E-mail: Rene.Schott@loria.fr 


\section{Décomposition approchée de mosaiques de Voronoï correspondant à des processus de Poisson modulés}

Résumé : Dans l'espace euclidien, nous considérons la mosaïque de Voronoï générée par un processus de Poisson non'homogène dont l'intensité prend différentes valeurs constantes sur les ensembles d'une certaine partition finie de l'espace. Considérant les cellules de Voronoï comme des marques associées à des points du processus ponctuel, nous montrons que la mesure intensité (mesure moyenne) du processus de Poisson ponctuel marqué admet une représentation par une formule de décomposition approchée. La valeur exacte est approximée par un mélange de mesures d'intensité correspondant à des modèles homogènes, tandis que la borne supérieure explicite du reste peut être calculée numériquement pour une large classe d'exemples pratiques. Des approximations du même type sont obtenues pour les distributions de Palm grâce à la formule de Campbell. L'analyse d'une large classe de mosaïques de Voronoï correspondant à des processus de Poisson ponctuels non-homogènes est possible grâce à des formules et des estimations déjà établies pour le cas homogène. Notre analyse s'applique aussi au processus de Poisson modulé par une partition aléatoire indépendante et stationnaire. Dans ce cas le terme d'erreur dépend de certaines formes intégrales définies sur les bords des éléments de la partition.

Mots-clés : $\quad$ Mosaïque de Voronoï, processus de Poisson ponctuel non-homogène, processus de Poisson ponctuel doublement stochastique, modèle booléen, approximations, décomposabilité. 


\section{Introduction}

Voronoi tessellation (VT) is a frequently used model of tessellation of the space (an extensive list of areas of applications can be found in [13, 12]). For a given locally finite system of points in the Euclidian space, VT is a division of the space into polyhedra (into polygons in the case of the plane) "about" the points of the system. Precisely, the Voronoi polygon (cell in common terminology) about a chosen point of the system is the subset of points of the space that lie closer to the chosen point than to any other point of the system. If the underlying system of points is a Poisson point process we call the resulting random tessellation the Poisson Voronoi tessellation (PVT).

In order to study statistical properties of random VT's one introduces the so called typical cell of the tessellation. Very roughly speaking, in stationary case, it can be seen as "randomly chosen" from the set of cells. In non-stationary case its distribution depends on the location and is interpreted as conditional, given the underlying process has its point at this location (formal definitions require Palm theory; see Section 2). Known formulae for distributional properties of the typical cell of PVT's are almost entirely confined to the stationary (homogeneous) case. Even then, formulae are very complicated and mainly approximations are known (see a review in Section 10.6 of [13], and [9, 8, 6, 4] for some new results).

In this paper we study an approximation technique for the distribution of the typical cell of VT's generated by some class of modulated-Poisson point processes. The idea is to approximate the unknown distribution in the non-homogeneous case by a mixture of the known distributions for homogeneous Poisson cases. We are able to give analytically tractable bounds for the error of the approximation in total variation. This approach makes possible the analysis of a wide class of nonhomogeneous PVT's by means of the formulae and estimates already established for homogeneous cases.

Specifically, we consider the Poisson point process whose intensity takes different constant values on sets of some finite partition of the space. Note that the cell of the VT about a given point is fully shaped by the neighbors of that point in the system of generating points. Thus, provided the partition of the space is not very "fine" with respect to the intensities of the points, the resulting modulated-Poisson Voronoi tessellation (mPVT) is "locally homogeneous" PVT. Consequently, the "typical cell of a given partitioning set" is highly probably identical to the typical cell of the homogeneous scenario and a "randomly chosen cell from the whole mPVT" should have a distribution close to the mixture of the homogeneous cases. The error of such approximation comes from existence of cells that cross the boundaries between the partitioning sets. In the paper we formalize the above intuitive approximations and quantify the errors. Our analysis applies also to the Poisson process modulated by an independent stationary random partition, in which case the error of the approximation of the double-stochastic-Poisson VT depends on some integrated linear contact distribution functions of the boundaries of the partition elements.

One of the motivations for the study in question is modeling of modern communication networks, where application of the PVT has already proven to give some interesting results (see eg. $[1,2,3,7])$. Generally speaking, within this setting points of the Poisson process represent various communication devices (concentrators, routers, base stations, etc.) and the associated cells represent the regions of the plane or space served by these devices. Adopting homogeneous scenarios in such models is often to simplistic, since it ignores spatial fluctuations of the traffic (large cities versus rural areas etc). On the other hand, more adequate, nonhomogeneous models rapidly become to difficult to analyze. A possible attitude to take if we 
want to improve upon this situation is to find a general framework, in which already available results concerning homogeneous cases could be integrated as "local solutions" into a "global" non-homogeneous model. We shall indicate that the paper demonstrates only very basic results for this approach, that could, however, help in working out of such a framework.

One can find in our approach yet another incarnation of a general idea of decomposability. This technique, that has been productive in analysis of economic, queueing and computer system models, relies on clustering of a large system of all variables into a small number of groups so that: (i) the interactions among the variables of each single group may be studied as if interactions among groups did not exit, and (ii) interactions among groups may be studied without reference to the interactions within groups. The system in this case is completely decomposable. When the interactions between groups of variables are non-null, but weak compared with interactions within groups, the system is said to be near-completely decomposable (see the monograph [5]). Using this terminology, our mPVT is near-completely decomposable model (more precisely: "near-completely decomposable in mean") and a modulated-Poisson Voronoi quasi-tessellation introduced in Example 3.3 is its completely decomposable approximation.

The rest of the paper is organized as follows. In Section 2 we introduce the modulated Poisson Voronoi tessellation and recall notions and facts concerning point processes, random closed sets and Palm theory that are our main tools. A general modulated marked point process, being our generic completely decomposable model, is introduced in Section 3; two basic results concerning the decomposability of its intensity and the distribution of its typical mark are given there too. The main results concerning the approximation decomposition of the modulatedPoisson Voronoi tessellation with deterministic and random modulation are given in Sections 4 and 5 respectively.

\section{Preliminaries}

In this section we introduce modulated Poisson Voronoi tessellation and recall notions and facts concerning point processes, random closed sets and Palm theory that are used throughout the paper.

\subsection{Modulated-Poisson Voronoi tessellation - formulation of the prob- lem}

Let $\Phi_{u}=\sum_{i} \varepsilon_{X_{i}^{u}}, u=1, \ldots, \ell$ be independent stationary Poisson point processes on $\mathbb{R}^{d}$, with intensities, respectively $\lambda_{u}>0$; here and throughout $\varepsilon_{z}$ is the atom measure at $z$. Let a measurable partition $\chi=\left\{\chi_{u}: u=1, \ldots, \ell\right\}$ of $\mathbb{R}^{d}$ be given (the basic measure theoretic details concerning the considered objects are given below). We call the inhomogeneous Poisson point process

$$
\Phi_{\chi} \equiv \sum_{u=1}^{\ell} \sum_{i} \mathbb{I}\left(X_{i}^{u} \in \chi_{u}\right) \varepsilon_{X_{i}^{u}}
$$

the $\chi$-modulated Poisson process $(\chi$-mod PP). Obviously $\chi$-mod PP is an (in general) inhomogeneous Poisson point process with intensity measure $\Lambda_{\chi}(\cdot)$ given by

$$
\Lambda_{\chi}(\mathrm{d} x) \equiv \mathbf{E}\left[\int_{\mathbb{R}^{d}} \mathbb{I}(y \in \mathrm{d} x) \Phi_{\chi}(\mathrm{d} y)\right]=\sum_{u=1}^{\ell} \mathbb{I}\left(x \in \chi_{u}\right) \lambda_{u} \mathrm{~d} x .
$$


Denote by $V\left(X_{i}, \Phi_{\chi}\right)$ the subset of points of $\mathbb{R}^{d}$ that lie closer to $X_{i}$ than to any other point of $\Phi_{\chi}$. The Voronoi tessellation

$$
\mathcal{V}\left(\Phi_{\chi}\right) \equiv \sum_{X_{i} \in \Phi_{\chi}} \varepsilon_{\left(X_{i}, V\left(X_{i}, \Phi_{\chi}\right)-X_{i}\right)}
$$

generated by $\Phi_{\chi}$ (the formal definition is given below) will be called the $\chi$-modulated-Poisson Voronoi tessellation $(\chi$-mod PVT). Note that we consider the Voronoi tessellation as a marked point process, with marks being cells translated to the origin $\left(C-x=\left\{y-x \in \mathbb{R}^{d}: y \in C\right\}\right)$, and as such, it has its intensity measure

$$
\tilde{\Lambda}_{\chi}^{v}(\mathrm{~d} x \times L) \equiv \mathbf{E}\left[\int_{\mathbb{R}^{d}} \mathbb{I}(y \in \mathrm{d} x) \mathbb{I}\left(V\left(0, \Phi_{\chi}-x\right) \in L\right) \Phi_{\chi}(\mathrm{d} y)\right],
$$

where $L$ is an appropriately measurable subset of the space of closed subsets of $\mathbb{R}^{d}$ (see the details bellow) and $\sum_{i} \varepsilon_{x_{i}}+x=\sum_{i} \varepsilon_{x_{i}+x}$.

Note at this stage, that this intensity does not admit any exact decomposition analogous to (2.1). A reason for this is that the Voronoi cell $V\left(x, \Phi_{\chi}\right)$ of a given point $x$ of the $\chi$-mod PP $\Phi_{\chi}$ depends on its neighboring points, which might be in different sets of the partition $\chi$. However, sometimes such decomposition might be a good approximation. In order to make this idea precise we need to recall some notions and introduce the notation.

\subsection{Measure-theoretic settings}

We consider the Euclidian space $\mathbb{R}^{d}$ with its Borel $\sigma$-algebra $\mathcal{B}\left(\mathbb{R}^{d}\right)$. A generic space of marks $\mathbb{M}$ is a separable, complete metric space (polish space) with its Borel $\sigma$-algebra $\mathcal{B}(\mathbb{M})$. A specific example of this space of marks, considered throughout this paper, is the space $\mathcal{F}$ of all closed subsets of $\mathbb{R}^{d}$. The topology on $\mathcal{F}$ is generated by the base of subsets $\{F \in \mathcal{F}: F \cap K=$ $\emptyset, F \cap O \neq \emptyset\}$ for all $K, O$, respectively, compact and open subsets of $\mathbb{R}^{d}$. The space $\mathcal{F}$ is compact, Hausdorf, and separable (see Theorem 1-2-1, p. 3 in [10]) and we consider its Borel $\sigma$-algebra $\mathcal{B}(\mathcal{F})$. Let $\tilde{\mathcal{N}}$ denote the space of counting measures on $\mathbb{R}^{d} \times \mathbb{M}$ that are bounded on $B \times \mathbb{M}$ for bounded $B \in \mathcal{B}\left(\mathbb{R}^{d}\right)$, with the $\sigma$-algebra $\sigma_{\tilde{\mathcal{N}}}$ on $\tilde{\mathcal{N}}$ generated by the mappings $\tilde{\mathcal{N}} \ni \tilde{\phi} \longmapsto \tilde{\phi}(B \times L)$ for all $B \in \mathcal{B}\left(\mathbb{R}^{d}\right), L \in \mathcal{B}(\mathbb{M})$. A marked point process $\tilde{\Phi}$ is a measurable mapping from a given probabilty space to $\tilde{\mathcal{N}}$. A non-marked point process $\Phi$ can be seen as $\tilde{\Phi}$ with a trivial one-element space of marks $\mathbb{M}$. A random closed set $\mathbb{X}$ is a measurable mapping from a given probability space to $\mathcal{F}$. For more details on measure-theoretic background of random closed sets see [10].

\subsection{Campbell formula}

Consider marked point processes $\tilde{\Phi}=\sum_{i} \varepsilon_{\left(X_{i}, M_{i}\right)}$ with points $\Phi_{u}=\sum_{i} \varepsilon_{X_{i}}$ located in $\mathbb{R}^{d}$ and with marks $M_{i}$ in a measurable polish space $\mathbb{M}$. We denote the intensity measure of $\tilde{\Phi}$ by $\tilde{\Lambda}(\mathrm{d}(x, m))$

$$
\tilde{\Lambda}(B, L) \equiv \mathbf{E}\left[\int_{\mathbb{R}}^{d} \int_{\mathbb{M}} \mathbb{I}(x \in B) \mathbb{I}(m \in L) \tilde{\Phi}(\mathrm{d}(x, m))\right]
$$

and by $\Lambda(\mathrm{d} x) \equiv \tilde{\Lambda}(\mathrm{d} x \times \mathbb{M})$ the respective intensity measure of points. Note that for fixed $L \in \mathcal{B}(\mathbb{M}), \tilde{\Lambda}(\cdot \times L)$ is absolutely continuous with respect to $\Lambda(\cdot)$ and thus we have the following 
disintegration called the Campbell formula

$$
\tilde{\Lambda}(B \times L)=\int_{\mathbb{R}^{d}} \int_{\mathbb{M}} \mathbb{I}(x \in B) \mathbb{I}(m \in L) \mathcal{M}(x ; \mathrm{d} m) \Lambda(\mathrm{d} x) .
$$

For fixed $x$, a (regular version) of the Radon-Nikodym derivative $\mathcal{M}(x ; \cdot)$ is called the (local) Palm distribution of the mark of the point at $x$, or, more loosely speaking, the distribution of the typical mark associated with the point located at $x$. (see e.g. [13]). If $\tilde{\Phi}$ is stationary (motion invariant); i.e, when the distribution of $\sum_{i} \varepsilon_{\left(X_{i}+x, M_{i}\right)}$ is the same as this of $\sum_{i} \varepsilon_{\left(X_{i}, M_{i}\right)}$ for all $x \in \mathbb{R}^{d}$, it is also true that

$$
\tilde{\Lambda}(B \times L)=\int_{\mathbb{R}^{d}} \int_{\mathbb{M}} \mathbb{I}(x \in B) \mathbb{I}(m \in L) \mathcal{M}(\mathrm{d} m) \Lambda(\mathrm{d} x) .
$$

where $\lambda$ is called the intensity (constant) of the point process, and $\mathcal{M}$ is the so called distribution of the typical mark.

We say that $\tilde{\Phi}$ is independently marked if (for any ordering of points that depends only on their locations) marks $\left\{M_{i}\right\}$ constitute a sequence of independent, identically distributed random elements. Then the distribution of the typical mark $\mathcal{M}$ coincides with the distribution of any $M_{i}$.

\subsection{Voronoi tessellation}

For a given non-marked point process $\Phi=\sum_{i} \varepsilon_{X_{i}}$, the Voronoi tessellation (VT) generated by $\Phi$ is the marked point process

$$
\tilde{\Phi}=\sum_{i} \varepsilon_{\left(X_{i}, V_{i}(\Phi)-X_{i}\right)}
$$

where marks are shifted to the origin random closed sets $V_{i}(\Phi)=V\left(X_{i}, \Phi\right)$ (called cells) of points in $\mathbb{R}^{d}$ that lie closer to the given point $X_{i}$ than to any other point of $\Phi$; i.e.,

$$
V(x, \phi)=\left\{y \in \mathbb{R}^{d}:|y-x| \leq \inf _{\phi \ni z \neq x}|y-z|\right\}
$$

where $|x|$ is the Euclidian norm in $\mathbb{R}^{d}$.

If the underlying point process $\Phi$ is Poisson we will call $\tilde{\Phi}$ the Poisson-Voronoi tessellation $(P V T)$. By the Slinyak's theorem, the distribution of the typical cell $\mathcal{M}^{v}(x ; \cdot)$ of a point located at $x$ in a general PVT coincides with the distribution of the set $V\left(x, \Phi+\varepsilon_{x}\right)-x=V\left(0, \Phi-x+\varepsilon_{0}\right)$; i.e., with the distribution of the shifted to the origin cell created by an extra point at $x$ added to the original realization of $\Phi$. For more details on the Voronoi tessellation see $[11,12]$.

\subsection{Random closed set}

For any random closed set $\mathbb{X}$ in $\mathbb{R}^{d}$ let $T(K)=\mathbf{P}(\mathbb{X} \cap K \neq 0)\left(K \subset \mathbb{R}^{d}\right.$, compact $)$ be the capacity functional of $\mathbb{X}$. For a stationary (motion invariant) random closed set $\mathbb{X}$ let $p$ denote its volume fraction; $p=\mathbf{P}(0 \in \mathbb{X})=T(\{0\})$.

A random closed set $\mathbb{X}$ is a (stationary) Boolean model $(B M)$ if

$$
\mathbb{X}=\bigcup_{i} C_{i}+Y_{i}
$$


where $\sum_{i} \varepsilon_{\left(Y_{i}, C_{i}\right)}$ is an independently marked (stationary) Poisson point process (of germs) on $\mathbb{R}^{d}$, with marks $C_{i}$ (called grains) being random closed sets (subsets of $\mathbb{R}^{d}$ ). Let $\tilde{\Phi}$ be stationary with intensity of germs $\beta$. Then, the capacity functional of the stationary BM $\mathbb{X}$ is

$$
T(K)=1-\exp \left[-\beta \int \nu(\check{C}+K) \mathcal{M}(\mathrm{d} C)\right]
$$

where $\mathcal{M}(\cdot)=\mathbf{P}\left(C_{0} \in \cdot\right)$ is the distribution of the so called typical grain of the BM, $\check{C}=$ $0-C=\{-z: z \in C\}$, and $\nu(\cdot)$ denotes the Lebesgue measure in $\mathbb{R}^{d}$. The volume fraction $p$ of the BM $\mathbb{X}$ is equal to $1-e^{-\beta \bar{c}}$ where $\bar{c}=\int \nu(C) \mathcal{M}(\mathrm{d} C)$ is the mean volume of the typical grain. Fore more details on the Boolean model and its applications see [13].

\subsection{Minor notation}

Let $\nu_{d}$ denote the volume of the $d$-dimensional unit ball. For $\left(x_{1}, \ldots, x_{d+1}\right) \in \mathbb{R}^{d}$ not lying on a common hyper-plane of dimension $d-1$, by $B\left(x_{1}, \ldots, x_{d+1}\right)$ we denote the open ball circumscribed on them. Let $B_{x}(r)$ be the $d$-dimensional ball centered at $x$ and with radius $r$. For $A \subset \mathbb{R}^{d}$, we denote by $A^{c}=\mathbb{R}^{d} \backslash A$ the complement of $A$ and $\# A$ is the cardinal number of $A$. Throughout the paper $\varepsilon_{x}$ is the atom measure $\varepsilon_{x}(A)=1$ if $x \in A$ and 0 otherwise.

\section{Modulated marked point process}

In this section we define a general modulated marked point process and give two basic results concerning the decomposability of its intensity measure and the distribution of its typical mark. In view of these results we see it as our generic completely decomposable model. As an incarnation of the generic modulated marked point process, in Example 3.3 we introduce a modulated-Poisson Voronoi quasi-tessellation that can be seen as the completely decomposable approximation of the modulated-Poisson Voronoi tessellation.

We construct the following $\chi$-modulated marked point process $\tilde{\Phi}_{\chi}$ out of (possibly dependent) stationary marked point process $\tilde{\Phi}^{u}=\sum_{i} \varepsilon_{\left(X_{i}^{u}, M_{i}^{u}\right)}, u=1, \ldots, \ell$ and a partition $\chi=\left\{\chi_{u}: u=1, \ldots, \ell\right\}$ of $\mathbb{R}^{d}$

$$
\tilde{\Phi}_{\chi}=\sum_{u=1}^{\ell} \sum_{i} \mathbb{I}\left(X_{i}^{u} \in \chi_{u}\right) \varepsilon_{\left(X_{i}^{u}, M_{i}^{u}\right)} .
$$

Lemma 3.1 The intensity measure $\tilde{\Lambda}_{\chi}$ of $\tilde{\Phi}_{\chi}$ is given by

$$
\tilde{\Lambda}_{\chi}(B \times L)=\sum_{u=1}^{\ell} \lambda_{u} \nu\left(B \cap \chi_{u}\right) \mathcal{M}_{u}(L)
$$

where $\lambda_{u}$ are intensities of points and $\mathcal{M}_{u}$ distributions of typical marks of the component processes $\Phi_{u}$ and $B$ and $L$ are measurable subsets of $\mathbb{R}^{d}$ and $\mathbb{M}$, respectively. 
Proof:

$$
\begin{aligned}
\tilde{\Lambda}_{\chi}(B \times L) & =\mathbf{E}\left[\int_{\mathbb{R}^{d}} \int_{\mathbb{M}} \mathbb{I}(x \in B) \mathbb{I}(m \in L) \tilde{\Phi}_{\chi}(\mathrm{d}(x, m))\right] \\
& =\sum_{u=1}^{\ell} \mathbf{E}\left[\int_{\mathbb{R}^{d}} \int_{\mathbb{M}} \mathbb{I}\left(x \in B \cap \chi_{u}\right) \mathbb{I}(m \in L) \tilde{\Phi}_{u}(\mathrm{~d}(x, m))\right] \\
& =\sum_{u=1}^{\ell} \lambda_{u} \nu\left(B \cap \chi_{u}\right) \mathcal{M}_{u}(L)
\end{aligned}
$$

since $\tilde{\Phi}_{u}, u=1, \ldots, \ell$ are stationary.

Of course $\tilde{\Phi}_{\chi}$ in general is not a stationary point process. Lets now suppose that the partition $\chi$ is a realization of a motion invariant, random partition $\Xi=\left\{\Xi_{u}: 1, \ldots, \ell\right\}$, independent of $\tilde{\Phi}_{u}, u=1, \ldots, \ell$. For each realization $\Xi=\chi$ we construct the point process $\tilde{\Phi}_{\chi}$ as described before and denote the resulting stationary-modulated marked point process by $\Phi_{\Xi}$. Obviously, the point process $\tilde{\Phi}_{\Xi}$ is stationary, hence its intensity measure $\tilde{\Lambda}_{(\Xi)}$ is of the form $\lambda_{(\Xi)} \mathrm{d} x \times$ $\left.\mathcal{M}_{(\Xi)}(\mathrm{d} m){ }^{1}\right)$.

Lemma 3.2 The distribution of the typical mark of the stationary-modulated marked point process $\tilde{\Phi}_{\Xi}$ is given by

$$
\mathcal{M}_{(\Xi)}(L)=\frac{1}{\lambda_{(\Xi)}} \sum_{u=1}^{\ell} \lambda_{u} p_{u} \mathcal{M}_{u}(L)
$$

where $p_{u}$ is the volume fraction of $\Xi_{u}$ and the constant intensity is

$$
\lambda_{(\Xi)}=\sum_{u=1}^{\ell} \lambda_{u} p_{u}
$$

Proof: Using Lemma 3.1 we have the intensity measure $\tilde{\Lambda}_{(\Xi)}$ of $\tilde{\Phi}_{\Xi}$

$$
\tilde{\Lambda}_{(\Xi)}(B \times L)=\sum_{u=1}^{\ell} \lambda_{u} \mathbf{E}\left[\nu\left(B \cap \Xi_{u}\right)\right] \mathcal{M}_{u}(L) .
$$

Now, for each stationary set $\Xi_{u}$

$$
\begin{aligned}
\mathbf{E}\left[\nu\left(B \cap \Xi_{u}\right)\right] & =\int_{B} \mathbf{E}\left[\mathbb{I}\left(x \in \Xi_{u}\right)\right] d x \\
& =\int_{B} \mathbf{E}\left[\mathbb{I}\left(0 \in \Xi_{u}\right)\right] d x \\
& =\nu(B) p_{u} .
\end{aligned}
$$

Applying this to (3.5) and normalizing we get (3.3) and (3.4).

Note that our $\chi$-mod PVT (2.2) is not a $\chi$-modulated marked point process in the sense of the definition (3.1). The difference will be clear in view of the following example.

\footnotetext{
${ }^{1}$ We use $(\Xi)$ as the subscript to emphasize that the respective expectations are taken with respect to the distribution of $\Xi$ too. Thus, $\mathcal{M}_{\Xi}$ is equal to conditional $\mathcal{M}_{(\Xi)}$ given the realization $\Xi$.
} 
Example 3.3 [A $\chi$-modulated-Poisson Voronoi quasi-tessellation]

Let $\mathcal{V}\left(\Phi_{u}\right)=\sum_{i} \varepsilon_{\left(X_{i}^{u}, V_{i}^{u}-X_{i}^{u}\right)}$ be PVT's generated by independent homogeneous Poisson point processes $\Phi_{u}$ with intensities $\lambda_{u}, u=1, \ldots, \ell$. We will call the following marked point process

$$
\mathcal{V}^{q}\left(\Phi_{\chi}\right)=\sum_{u=1}^{\ell} \sum_{i} \mathbb{I}\left(X_{i}^{u} \in \chi_{u}\right) \varepsilon_{\left(X_{i}^{u}, V_{i}^{u}-X_{i}^{u}\right)}
$$

the $\chi$-modulated-Poisson Voronoi quasi-tessellation $(\chi$-mod PVqT). Note that the $\chi$-mod PVqT $\mathcal{V}^{q}\left(\Phi_{\chi}\right)$ is a modulated marked point process in the sense of the definition (3.1) thus properties of its cells can be established by means of Lemmas 3.1 and 3.2. On the other hand, the cells of our quasi-tessellation, unlike cells of a "true tessellation", might not be disjoint and their union might not cover the whole space. Obviously the phenomena of "overlapping" cells and "wholes" are more likely to occur close to the boundary of each $\chi_{u}$ where some points of $\Phi_{u}$ have neighbors among the points of $\Phi_{v}, v \neq u$. It would be a true tessellation, namely the $\chi$-mod PVT, if all the cells were generated by the common pattern $\Phi_{\chi}$ of points and not out of the component point processes $\Phi_{u}$. However, we might expect, that at least in some cases, the existence of the cells intersecting boundaries of $\chi_{u}$ is negligible and the distribution of the "true" $\chi$-mod PVT can be approximated by $\chi$-mod PVqT. This idea will be developed in the remaining part of the paper.

\section{Decomposition approximation of the modulated-Poisson Voronoi tessellation}

In this section we show how the intensity measure of the modulated-Poisson Voronoi tessellation can be approximated by the intensity measure of the modulated-Poisson Voronoi quasitessellation of Example 3.3 that admits the complete decomposition given in Lemma 3.1. In Proposition 4.2, that is our main result concerning approximations of the model with the deterministic modulation $\chi$, we give the upper bound for the error of the approximation. As a corollary we obtain an approximate decomposition of the distribution of the typicall cell located at a given point. The quality of the approximation depends on some integrated distance of this point to the boundary of the element of the partition it belongs to.

Lets denote by $\tilde{\Lambda}_{\chi}^{v}$ the intensity measure of the $\chi$-mod PVT $\mathcal{V}\left(\Phi_{\chi}\right)$ and let $\mathcal{M}_{u}^{v}$ by the distribution of the typical mark (cel) of the homogeneous $\operatorname{PVT} \mathcal{V}\left(\Phi_{u}\right), u=1, \ldots, \ell$.

For each $u=1, \ldots, \ell$ lets define an auxiliary dominating process

$$
\Phi_{u}^{\max }=\sum_{u^{\prime}: \lambda_{u^{\prime}} \geq \lambda_{u}} \sum_{i} \mathbb{I}\left(X_{i}^{u^{\prime}} \in \chi_{u^{\prime}}\right) \varepsilon_{X_{i}^{u^{\prime}}}+\sum_{u^{\prime \prime}: \lambda_{u^{\prime \prime}}<\lambda_{u}} \sum_{i} \mathbb{I}\left(X_{i}^{u} \in \chi_{u^{\prime \prime}}\right) \varepsilon_{X_{i}^{u}} .
$$

For a given point $x \in \mathbb{R}^{d}$ and a realization $\phi$ of a point process on $\mathbb{R}^{d}$ let $\mathcal{N}(x, \phi)$ denote the subset of points of $\phi$, which in the Voronoi tesselation $\mathcal{V}\left(\phi+\varepsilon_{x}\right)$ have cells sharing an edge with the cell $V\left(x, \phi+\varepsilon_{x}\right)$. Formally

$$
\mathcal{N}(x, \phi)=\left\{y \in \phi: \phi\left(B\left(x, y, z_{1}, \ldots, z_{d-1}\right)\right)=0 \text { for some } z_{1}, \ldots, z_{d-1} \in \phi\right\} .
$$

Finally, let

$$
\zeta(\chi, \phi)=\bigcup_{u=1}^{\ell}\left\{x \in \chi_{u}: \mathcal{N}(x, \phi) \cap \chi_{u}^{c} \neq \emptyset\right\}
$$

$\mathrm{RR} \mathrm{n}^{\circ} 4585$ 
The following lemma gives an approximate decomposition of the intensity measure of $\chi$ $\bmod$ PVT. Note that the sum $\sum_{u=1}^{\ell}$ in (4.1) bellow is the intensity measure of the $\chi$-modulatedPoisson Voronoi quasi-tessellation from Example 3.3.

Lemma 4.1 The intensity measure $\tilde{\Lambda}_{\chi}^{v}$ of $\chi$-mod $P V T \mathcal{V}\left(\tilde{\Phi}_{\chi}\right)$ admits the following decomposition

$$
\tilde{\Lambda}_{\chi}^{v}(B \times L)=\sum_{u=1}^{\ell} \lambda_{u} \nu\left(B \cap \chi_{u}\right) \mathcal{M}_{u}^{v}(L)+R(B \times L),
$$

where $B$ and $L$ are measurable subsets of $\mathbb{R}^{d}$ and $\mathbb{M}$ respectively, and the remaining term is equal to

$$
\begin{aligned}
& R(B \times L) \\
& \quad=\sum_{u=1}^{\ell} \mathbf{E}\left[\int_{\chi_{u} \cap B} \mathbb{I}\left(x \in \zeta\left(\chi, \Phi_{u}^{\max }\right)\right)\left(\mathbb{I}\left(V\left(0, \Phi_{\chi}-x\right) \in L\right)-\mathbb{I}\left(V\left(0, \Phi_{u}-x\right) \in L\right)\right) \Phi_{\chi}(\mathrm{d} x)\right] .
\end{aligned}
$$

Proof: Note first that we can take the probability space such that almost surely $\Phi_{u_{1}} \subset \Phi_{u_{2}} \subset$ $\ldots \subset \Phi_{u_{\ell}}$, where $\lambda_{u_{1}} \leq \lambda_{u_{2}} \leq \ldots \leq \lambda_{u_{\ell}}$. In fact, the distribution of the $\Phi_{\chi}$ remains the same if all $\Phi_{u}$ are constructed by successive thinning of $\Phi_{u_{\ell}}$. Let it be the case. Following along the same line as in the proof of Lemma 3.1 we state that (4.1) is true with the remaining term equal to

$$
\sum_{u=1}^{\ell} \mathbf{E}\left[\int_{\chi_{u} \cap B} \mathbb{I}\left(V\left(x, \Phi_{\chi}\right) \neq V\left(0, \Phi_{u}\right)\right)\left(\mathbb{I}\left(V\left(0, \Phi_{\chi}-x\right) \in L\right)-\mathbb{I}\left(V\left(0, \Phi_{u}-x\right) \in L\right)\right) \Phi_{\chi}(\mathrm{d} x)\right] .
$$

Note now that $V\left(x, \Phi_{\chi}\right)=V\left(x, \Phi_{u}\right)$ is equivalent to $\mathcal{N}\left(x, \Phi_{\chi}\right)=\mathcal{N}\left(x, \Phi_{u}\right)$. Moreover, for $x \in$ $\chi_{u}$, if $\mathcal{N}\left(x, \Phi_{u}^{\max }\right) \subset \chi_{u}$, then $\mathcal{N}\left(x, \Phi_{u}^{\max }\right)=\mathcal{N}\left(x, \Phi_{\chi}\right)=\mathcal{N}\left(x, \Phi_{u}\right)$. Thus $V\left(x, \Phi_{\chi}\right) \neq V\left(x, \Phi_{u}\right)$ implies $\mathcal{N}\left(x, \Phi_{u}^{\max }\right) \not \subset \chi_{u}$; i.e., $x \in \zeta\left(\chi, \Phi_{u}^{\max }\right)$, which completes the proof.

Our main task now is to give a numerically tractable upper bound for the absolute value of the correcting term $R(\cdot, \cdot)$ in the decomposition (4.1). Lets denote by $\lambda_{\max }=\max \left\{\lambda_{u}: u=\right.$ $1, \ldots, \ell\}, \lambda_{\min }=\min \left\{\lambda_{u}: u=1, \ldots, \ell\right\}$.

Proposition 4.2 The intensity measure $\tilde{\Lambda}_{\chi}^{v}$ admits the docomposition (4.1) and the remaining term is bounded by

$$
\begin{aligned}
& |R(B \times L)| \\
& \quad \leq \frac{2^{d^{2}}}{d} \sum_{u=1}^{\ell} \frac{\lambda_{\max }^{d-1}}{\lambda_{u}^{d-2}} \int_{\mathbb{R}^{d}} \int_{\mathbb{R}^{d}} e^{-\lambda_{u} \nu_{d}|x-y|^{d} / 2^{d+1}} \mathbb{I}\left(x \in \chi_{u} \cap B\right)\left(\sum_{u^{\prime} \neq u} \max \left(\lambda_{u}, \lambda_{u^{\prime}}\right) \mathbb{I}\left(y \in \chi_{u^{\prime}}\right)\right) \mathrm{d} x \mathrm{~d} y .
\end{aligned}
$$

Proof: Using Lemma 4.1

$$
|R(B \times L)| \leq \sum_{u=1}^{\ell} 2 \mathbf{E}\left[\int_{\chi_{u}} \mathbb{I}(x \in B) \mathbb{I}\left(x \in \zeta\left(\chi, \Phi_{u}^{\max }\right)\right) \Phi_{\chi}(\mathrm{d} x)\right] .
$$

Note that by the definition

$$
\left.\mathbb{I}\left(x \in \zeta\left(\chi, \Phi_{u}^{\max }\right)\right)=\sum_{u=1}^{\ell} \mathbb{I}\left(x \in \chi_{u}, \mathcal{N}\left(x, \Phi_{u}^{\max }\right) \cap \chi_{u}^{c} \neq \emptyset\right)\right)
$$


and for $x \in \chi_{u}$, such that $\Phi_{u}(\{x\})=1$

$$
\begin{aligned}
& \mathbb{I}\left(\mathcal{N}\left(x, \Phi_{u}^{\max }\right) \cap \chi_{u}^{c} \neq \emptyset\right) \leq \#\left(\mathcal{N}\left(x, \Phi_{u}^{\max }\right) \cap \chi_{u}^{c}\right) \\
& \leq \frac{1}{d(d-1) !} \int_{\mathbb{R}^{d}} \mathbb{I}\left(y \in \chi_{u}^{c}\right) \overbrace{\int_{\mathbb{R}^{d}}^{d} \ldots \int_{\mathbb{R}^{d}}^{d-1}} \mathbb{I}\left(\Phi_{u}^{\max }\left(B\left(x, y, z_{1}, \ldots, z_{d-1}\right)\right)=0\right) \\
& \times\left(\Phi_{u}^{\max }\right)^{(d)}\left(\mathrm{d}\left(y, z_{1}, \ldots, z_{d-1}\right)\right),
\end{aligned}
$$

where

$$
\left(\Phi_{u}^{\max }\right)^{(d)}\left(\mathrm{d}\left(y, z_{1}, \ldots, z_{d-1}\right)\right) \mathbb{I}\left(y, z_{1}, \ldots, z_{d-1} \text { distinct }\right) \Phi_{u}^{\max }(\mathrm{d} y) \Phi_{u}^{\max }\left(\mathrm{d} z_{1}\right) \ldots \Phi_{u}^{\max }\left(\mathrm{d} z_{d-1}\right)
$$

is the so-called factorial moment of $\Phi_{u}^{\max }$ (note that $1 /(d(d-1) !)$ stands in the previous bound since each point $y \in \mathcal{N}\left(x, \Phi_{u}^{\max }\right)$, as a neighbour of $x$, is counted by the integral there with $d$ different tuples of co-neighbours $\left\{z_{1}, \ldots, z_{d-1}\right\}$ and each such non-ordered tuple is counted by the factorial moment $(d-1)$ ! times). This makes

$$
\begin{aligned}
& |R(B \times L)| \\
& \leq \sum_{u=1}^{\ell} \frac{2 \lambda_{\max }^{d-1} \lambda_{u}}{d !} \int_{\mathbb{R}^{d}} \mathbb{I}\left(x \in B \cap \chi_{u}\right) \int_{\mathbb{R}^{d}}\left(\sum_{u^{\prime} \neq u} \max \left(\lambda_{u}, \lambda_{u^{\prime}}\right) \mathbb{I}\left(y \in \chi_{u^{\prime}}\right)\right) \\
& \quad \times \int_{\mathbb{R}^{d}} \ldots \int_{\mathbb{R}^{d}} e^{-\lambda_{u} \nu\left(B\left(x, y, z_{1}, \ldots, z_{d-1}\right)\right)} \mathrm{d} x \mathrm{~d} y \mathrm{~d} z_{1} \ldots \mathrm{d} z_{d-1} .
\end{aligned}
$$

Next, we use approximation of the volume of the $d$-dimensional ball circumscribed on $x, y, z_{1}$, $\ldots, z_{d-1}$

$$
\begin{aligned}
\nu\left(\left(B^{o}\left(x, y, z_{1}, \ldots, z_{d-1}\right)\right)\right. & \geq \frac{\nu_{d}}{2^{d}}\left(\max _{i=1, \ldots, d-1}\left\{|x-y|,\left|y-z_{i}\right|\right\}\right)^{d} \\
& \geq \frac{\nu_{d}}{2^{d+1}}\left(|x-y|^{d}+\left(\max _{i=1, \ldots, d-1}\left|y-z_{i}\right|\right)^{d}\right) .
\end{aligned}
$$

Thus,

$$
\begin{aligned}
& \int_{\mathbb{R}^{d}} \ldots \int_{\mathbb{R}^{d}} e^{-\lambda_{u} \nu\left(B^{o}\left(x, y, z_{1}, \ldots, z_{d-1}\right)\right)} \mathrm{d} z_{1} \ldots \mathrm{d} z_{d-1} \\
& \quad \leq(d-1) ! e^{-\lambda_{u} \nu_{d}|x-y|^{d} / 2^{d+1}} \int_{\mathbb{R}^{d}} \ldots \int_{\mathbb{R}^{d}} e^{-\lambda_{u} \nu_{d}\left|z_{1}\right|^{d} / 2^{d+1}} \mathbb{I}\left(\left|z_{1}\right| \geq \ldots \geq\left|z_{d-1}\right|\right) \mathrm{d} z_{1} \ldots \mathrm{d} z_{d-1} \\
& \quad=(d-1) ! e^{-\lambda_{u} \nu_{d}|x-y|^{d} / 2^{d+1}} \frac{2^{(d+1)(d-1)}}{\lambda_{u}^{d-1}}
\end{aligned}
$$

and applying this to (4.4) we obtain (4.3).

Corollary 4.3 Let $\chi$-mod $P P \Phi_{\chi}$ be given, fix $x$ in the interior of $\chi_{u}$ for some $u \in\{1, \ldots, \ell\}$ and consider $\Phi_{\chi}+\varepsilon_{x}$. Then the distribution of the cell $V\left(x, \Phi_{\chi}+\varepsilon_{x}\right)$ can be approximated in 
total variation by the distribution of the typical cell $\mathcal{M}_{u}^{v}$ of the homogeneous $P V T \mathcal{V}\left(\Phi_{u}\right)$ as follows: for almost all $x \in \chi_{u}$

$$
\begin{aligned}
& \sup _{L \in \mathbb{M}}\left|\mathbf{P}\left(V\left(x, \Phi_{\chi}+\varepsilon_{x}\right)-x \in L\right)-\mathcal{M}_{u}^{v}(L)\right| \\
& \leq \frac{2^{d^{2}}}{d} \frac{\lambda_{\max }^{d-1}}{\lambda_{u}^{d-1}} \sum_{u^{\prime} \neq u} \max \left(\lambda_{u}, \lambda_{u^{\prime}}\right) \int_{\mathbb{R}^{d}} e^{-\lambda_{u} \nu_{d}|x-y|^{d} / 2^{d+1}} \mathbb{I}\left(y \in \chi_{u^{\prime}}\right) \mathrm{d} y .
\end{aligned}
$$

Proof: By the Slivnyak's theorem, we have for almost all $x$

$$
\mathbf{P}\left(V\left(x, \Phi_{\chi}+\varepsilon_{x}-x\right) \in L\right)=\frac{\mathrm{d} \tilde{\Lambda}_{\chi}(\cdot \times L)}{\mathrm{d} \Lambda_{\chi}(\cdot)}(x) .
$$

Thus, by Campbell formulas 2.3 and 2.4 and Proposition 4.2 for all measurable $B \subset \chi_{u}$

$$
\begin{aligned}
& \left|\int_{B} \mathbf{P}\left(V\left(x, \Phi_{\chi}+\varepsilon_{x}\right)-x \in L\right)-\mathcal{M}_{u}^{v}(L) \mathrm{d} x\right| \\
& \quad=\left|\frac{1}{\lambda_{u}} \tilde{\Lambda}_{\chi}^{v}(B \times L)-\nu(B) \mathcal{M}_{u}^{v}(L)\right| \\
& \quad \leq \frac{2^{d^{2}}}{d} \frac{\lambda_{\max }^{d-1}}{\lambda_{u}^{d-1}} \sum_{u^{\prime} \neq u} \max \left(\lambda_{u}, \lambda_{u^{\prime}}\right) \int_{\mathbb{R}^{d}} \int_{\mathbb{R}^{d}} e^{-\lambda_{u} \nu_{d}|x-y|^{d} / 2^{d+1}} \mathbb{I}(x \in B) \mathbb{I}\left(y \in \chi_{u^{\prime}}\right) \mathrm{d} x \mathrm{~d} y .
\end{aligned}
$$

This completes the proof, since $B$ and $L$ can be chosen arbitrarily.

The following result concerning means of bounded functionals of the Voronoi cell follows immediately from Corollary 4.3.

Corollary 4.4 Let $V\left(x, \Phi_{\chi}+\varepsilon_{x}\right)$ be as in Corollary 4.3 and let $f(\cdot)$ be a bounded set-function with $\sup _{\mathbb{R}^{d} \supset D \text { closed }}|f(D)| \leq f_{\text {sup }}<\infty$ for some constant $f_{\text {sup }}$. Then the mean function $f$ of the cell $V\left(x, \Phi_{\chi}+\varepsilon_{x}\right)$ can be approximated by the mean function $f$ of the typical cell of the homogeneous $P V T \mathcal{V}\left(\Phi_{u}\right)$ as follows

$$
\mathbf{E}\left[f\left(V\left(x, \Phi_{\chi}+\varepsilon_{x}\right)\right)\right]=\mathbf{E}\left[f\left(V\left(x, \Phi_{u}+\varepsilon_{x}\right)\right)\right]+f_{\text {sup }} R,
$$

where $|R|$ is upper-bounded by the right-hand-side of (4.5).

We shall show now two examples where the right-hand-side of (4.5) can be numerically calculated.

Example 4.5 [Cell located at some distance to a "hot/cold spot"] Let $\chi_{1}=B_{-r}(r), \chi_{2}=$ $\left.\mathbb{R}^{d} \backslash \chi_{1}\right)$ and $x \in \chi_{2}$. Then

$$
\begin{aligned}
|R| & \leq \frac{2^{d^{2}}}{d} \frac{\lambda_{\max }^{d}}{\lambda_{2}^{d-1}} \int_{\mathbb{R}^{d}} e^{-\lambda_{2} \nu_{d}|x-y|^{d} / 2^{d+1}} \mathbb{I}\left(y \in B_{-r}(r)\right) \mathrm{d} y \\
& =\frac{2^{d^{2}} \nu_{d}}{\pi} \frac{\lambda_{\max }^{d}}{\lambda_{2}^{d-1}} \int_{|x|}^{|x|+2 r} u^{d-1} \arccos \frac{(r+|x|)^{2}+u^{2}-r^{2}}{2 u(r+|x|)} e^{-\lambda_{2} \nu_{d} u^{d} / 2^{d+1}} d u .
\end{aligned}
$$

Figure 1 shows the above approximation calculated for $\lambda_{1}=5, \lambda_{2}=10$ ("cold spot") and $\lambda_{1}=10, \lambda_{2}=5$ ("hot spot"); $r=1$. 

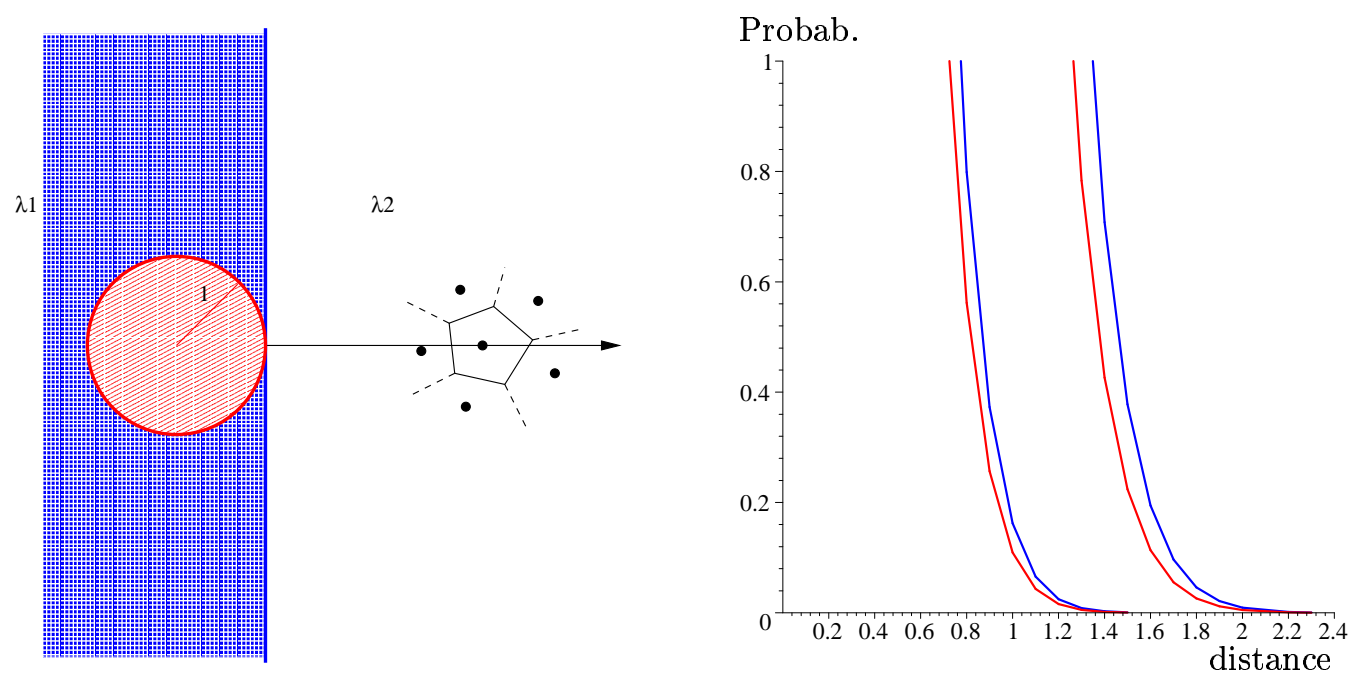

Figure 1: The upper bound for the probability that the region of different intensity of points in a Poisson point process has any impact on the Voronoi cell located at some distance to that region (see Examples 4.5, 4.6); curves from letf to right: cold spot, cold wall, hot spot, hot wall. Note a short-distance dependence of the region of inhomogeneity on the typical cell.

Example 4.6 [Cell located at some distance to a "hot/cold wall"] Let $\chi_{1}=$ be a halfspace, $\chi_{2}=\mathbb{R}^{d} \backslash \chi_{1}$ and the distance of $x \in \chi_{2}$. Then

$$
|R| \leq \frac{2^{d^{2}} \nu_{d}}{\pi} \frac{\lambda_{\max }^{d}}{\lambda_{2}^{d-1}} \int_{|x|}^{\infty} u^{d-1} \arccos \frac{|x|}{u} e^{-\lambda_{2} \nu_{d} u^{d} / 2^{d+1}} d u
$$

Figure 1 shows the above approximation calculated for $\lambda_{1}=5, \lambda_{2}=10$ ("cold wall") and $\lambda_{1}=10, \lambda_{2}=5$ ("hot wall").

\section{Decomposition approximation of the stationary modulated-Poisson Voronoi tessellation}

In this section we consider a stationay random partition of the space that modulates intensity of the, independent of it, Poisson process. We show how the distribution of the typical cell of the resulting stationary-modulated-Poisson Voronoi tessellation can be approximated by the distribution of the typical cell of the stationary version of the modulated-Poisson Voronoi quasi-tessellation of Example 3.3 that admits the complete decomposition of Lemma 3.2. In Proposition 5.1, that is our main result concerning approximations of the model with the random modulation, we give the upper bound for the error of the approximation. As a corollary we obtain an approximate decomposition of the distribution of the typical cell given its generating point is covered by some particular element of the partition. This time the quality of the approximation depends on some integrated measure of the total boundary of the partition. This measure can be interpreted in terms of the linear contact distribution functions of the boundaries of the elements of the partition. 
In this section we assume a stationary decomposing random partition $\Xi=\left\{\Xi_{u}: u=\right.$ $1, \ldots, \ell\}$; that is, that for any vector $x \in \mathbb{R}^{d}$ the distribution of $\Xi+x=\left\{\Xi_{u}+x: u=1, \ldots, \ell\right\}$ is the same as $\Xi$. Moreover, let $\Xi$ be independent of Poisson processes $\Phi_{u}, u=1, \ldots, \ell$. This makes the $\Xi$-mod PP $\Phi_{\Xi}$ the stationary double-stochastic-Poisson (Cox) point process and the stationary-Cox Voronoi tessellation (CoxVT) $\mathcal{V}\left(\Phi_{\Xi}\right)$ admits the distribution of the typical cell $\mathcal{M}_{(\Xi)}^{v}$.

Denote by $T_{\partial \Xi}^{\Sigma}(\cdot)$ the half of the sum of the capacity functionals of the boundaries of the elements $\Xi$; i.e., for each compact set $K \subset \mathbb{R}^{d}$

$$
T_{\partial \Xi}^{\Sigma}(K)=\frac{1}{2} \sum_{u=1}^{\ell} \mathbf{P}\left(\partial \Xi_{u} \cap K \neq \emptyset\right)=\frac{1}{2} \sum_{u=1}^{\ell} T_{\partial \Xi_{u}}(K),
$$

where $\partial \Xi_{u}$ is the boundary of $\Xi_{u}$. Let $p_{u}=\mathbf{P}\left(0 \in \Xi_{u}\right)$ denote the volume fraction of $\Xi_{u}$. For any $x, y \in \mathbb{R}^{d}$ let $\langle x, y\rangle=\left\{z \in \mathbb{R}^{d}: z=\xi x+(1-\xi) y, \xi \in[0,1]\right\}$ be the segment in $\mathbb{R}^{d}$.

The following proposition gives an approximate decomposition of the distribution of the typical cell of $\chi$-mod PVT. Note that the sum $\sum_{u=1}^{\ell}$ in (5.1) below is the distribution of the typical cell of the $\Xi$-modulated-Poisson Voronoi quasi-tessellation (cf Example 3.3).

Proposition 5.1 The distribution of the typical mark of the stationary-Cox Voronoi tessellation $\mathcal{V}\left(\Phi_{\Xi}\right)$ admits the following decomposition

$$
\mathcal{M}_{(\Xi)}^{v}(L)=\frac{1}{\lambda_{(\Xi)}} \sum_{u=1}^{\ell} \lambda_{u} p_{u} \mathcal{M}_{u}^{v}(L)+R
$$

where $\lambda_{(\Xi)}=\sum_{u=1}^{\ell} \lambda_{u} p_{u}$ and the remainder term is bounded by

$$
|R| \leq \frac{1}{\lambda_{(\Xi)}} \frac{2^{d^{2}}}{d} \frac{\lambda_{\max }^{d}}{\lambda_{\min }^{d-2}} \int_{\mathbb{R}^{d}} e^{-\lambda_{\min \nu_{d}}|y|^{d} / 2^{d+1}} T_{\partial \Xi}^{\Sigma}(\langle 0, y\rangle) \mathrm{d} y .
$$

Proof: Use Proposition 4.2 and observe that

$$
\begin{aligned}
\mathbf{E}\left[\sum_{u=1}^{\ell} \mathbb{I}\left(x \in \Xi_{u} \cap B\right) \mathbb{I}\left(y \in \Xi_{u}^{c}\right)\right] & \leq \frac{1}{2} \mathbb{I}(x \in B) \sum_{u=1}^{\ell} \mathbf{P}\left(\partial \Xi_{u} \cap\langle x, y\rangle \neq \emptyset\right) \\
& =\mathbb{I}(x \in B) T_{\partial \Xi}^{\Sigma}(\langle x, y\rangle) .
\end{aligned}
$$

By the stationarity of $\Xi$

$$
\begin{aligned}
& \int_{\mathbb{R}^{d}} \int_{\mathbb{R}^{d}} e^{-\lambda_{\min } \nu_{d}|x-y|^{d} / 2^{d+1}} \mathbb{I}(x \in B) T_{\partial \Xi}^{\Sigma}(\langle x, y\rangle) \mathrm{d} x \mathrm{~d} y \\
& \quad=\nu(B) \int_{\mathbb{R}^{d}} e^{-\lambda_{\min } \nu_{d}|y|^{d} / 2^{d+1}} T_{\partial \Xi}^{\Sigma}(\langle 0, y\rangle) \mathrm{d} y,
\end{aligned}
$$

which completes the proof.

Note that the functional $T_{\partial \Xi}^{\Sigma}(\langle 0, y\rangle)$ can be interpreted also in terms of the so called linear contact distribution functions (lcdf's) of the boundaries of the elements $\partial \Xi_{u}$ of the partition $\Xi$. For a given unit interval $\langle 0, \mathbf{e}\rangle,|\mathbf{e}|=1$, the lcdf of $\partial \Xi_{u}$ is

$$
H_{\langle 0, \mathbf{e}\rangle}^{u}(t)=1-\frac{1-T_{\partial \mathbb{X}}(\langle 0, t \mathbf{e}\rangle)}{1-T_{\partial \mathbb{X}}(\{0\})}, \quad t \geq 0
$$


(cf [13], section 3.1.1, p. 71).

The following result concerning mean bounded functionals of the Voronoi cell follows immediately from Proposition 5.1.

Corollary 5.2 Let $V\left(0, \Phi_{\Xi}+\varepsilon_{0}\right)$ be the typical cell of the CoxVT $\mathcal{V}\left(\Phi_{\Xi}\right)$ as in Proposition 5.1 and let $f(\cdot)$ be a bounded set-function with $\sup _{\mathbb{R}^{d} \supset D \text { closed }}|f(D)| \leq f_{\text {sup }}<\infty$ for some constant $f_{\text {sup }}$. Then the mean function $f$ of the cell $V\left(0, \Phi_{\Xi}+\varepsilon_{0}\right)$ can be approximated by the mixture of the means in homogeneous $P V T$ 's $\mathcal{V}\left(\Phi_{u}\right)$ as follows

$$
\mathbf{E}\left[f\left(V\left(0, \Phi_{\Xi}+\varepsilon_{0}\right)\right)\right]=\frac{1}{\lambda_{(\Xi)}} \sum_{u=1}^{\ell} \lambda_{u} p_{u} \mathbf{E}\left[f\left(V\left(0, \Phi_{u}+\varepsilon_{0}\right)\right)\right]+f_{\text {sup }} R,
$$

where $R$ satisfies (5.2).

In a similar manner we can prove the following approximation of the distribution of the typical cell, given its generator is covered by a particular element $\Xi_{u}$ of the partition $\Xi$. This distribution formally can be defined as the distribution $\mathcal{M}_{\left(\Xi \Xi_{u}\right)}^{v}$ of the typical mark of the following stationary marked point process

$$
\sum_{X_{i} \in \Phi_{\Xi}} \mathbb{I}\left(X_{i} \in \Xi_{u}\right) \varepsilon_{\left(X_{i}, V_{i}\left(\Phi_{\Xi}\right)-X_{i}\right)}
$$

Proposition 5.3 Fix $u \in\{1, \ldots, \ell\}$. The distribution of the typical mark of the stationary-Cox Voronoi tessellation $\mathcal{V}\left(\Phi_{\Xi}\right)$ given its generator is covered by $\Xi_{u}$ admits the following decomposition

$$
\mathcal{M}_{\left(\Xi \mid \Xi_{u}\right)}^{v}(L)=\frac{p_{u} \lambda_{u}}{\lambda_{(\Xi)}} \mathcal{M}_{u}^{v}(L)+R
$$

where $\lambda_{(\Xi)}=\sum_{u=1}^{\ell} \lambda_{u} p_{u}$ and the remainder term is bounded

$$
|R| \leq \frac{1}{\lambda_{(\Xi)}} \frac{2^{d^{2}}}{d} \frac{\lambda_{\max }^{d}}{\lambda_{\min }^{d-2}} \int_{\mathbb{R}^{d}} e^{-\lambda_{\min } \nu_{d}|y|^{d} / 2^{d+1}} T_{\partial \Xi_{u}}(\langle 0, y\rangle) \mathrm{d} y
$$

Proof: It goes along the same lines as the proof of Proposition 5.3.

Example 5.4 [Boolean-modulated-Poisson Voronoi tessellation (BmPVT)] Let the partition $\Xi=\left\{\mathbb{X}, \mathbb{X}^{c}\right\}$ be given, where $\mathbb{X}$ is a stationary Boolean Model (2.5). Note that

$$
T_{(\Xi)}=\frac{1}{2}\left(\mathbf{P}(\partial \mathbb{X} \cap K \neq 0)+\mathbf{P}\left(\partial \mathbb{X}^{c} \cap K \neq 0\right)\right)=T_{\partial \mathbb{X}}(K)
$$

and that $\partial \mathbb{X} \subset \mathbb{X}^{\prime} \equiv \bigcup_{i} \partial C_{i}+Y_{i}$ is another BM. Thus, using the general formula (2.6) for the capacity functional of a $\mathrm{BM}$, we get

$$
T_{\partial \Xi}^{\Sigma}(\langle 0, y\rangle) \leq T_{\left(\mathbb{X}^{\prime}\right)}(\langle 0, y\rangle)=1-\exp \left\{-\beta \mathbf{E}\left[\nu\left(\left(\partial \check{C}_{0}\right)+\langle 0, y\rangle\right)\right]\right\} .
$$

In the following we assume that $C_{0}$ is the ball $B_{0}(\rho)$ with a random radius $\rho$. Then $\partial B_{0}(\rho)=$ $S_{0}(\rho)$ is the respective sphere and thus

$$
\nu\left(\left(\partial \check{C}_{0}\right)+\langle 0, y\rangle\right)=\nu\left(S_{0}(\rho)+\langle 0, y\rangle\right) \leq 2 d \nu_{d} \rho^{d-1}|y|
$$

$\operatorname{RR} n^{\circ} 4585$ 

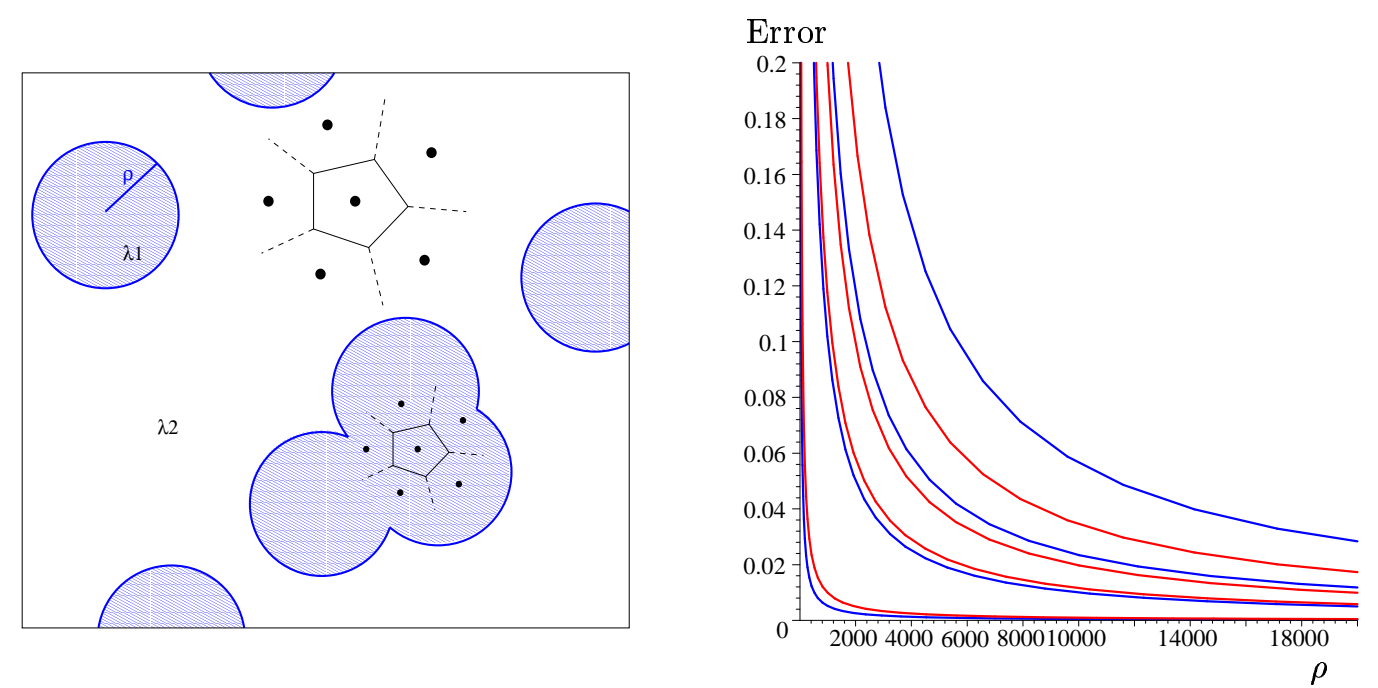

Figure 2: Error of the approximation of the typical Voronoi cell distribution in Boolean-modulated-Poisson model by the appropriate mixture of Poisson Voronoi models (see Example 5.4); curves correspond to different (constant) volume fractions of the Boolean Model with varying radius $\rho$ of its individual ball.

Recall that $p_{1}=1-p_{2}=1-\exp \left[-\beta \nu_{d} \mathbf{E}\left[\rho^{d}\right]\right]$ and thus

$$
\lambda_{(\Xi)}=\lambda_{1}-\left(\lambda_{1}-\lambda_{2}\right) \exp \left[-\beta \nu_{d} \mathbf{E}\left[\rho^{d}\right]\right] \text {. }
$$

Using (5.2), we have for our BmPVT

$$
\begin{aligned}
|R| & \leq \frac{1}{\lambda_{(\Xi)}} \frac{2^{d^{2}}}{d} \frac{\lambda_{\max }^{d}}{\lambda_{\min }^{d-2}} \int_{\mathbb{R}^{d}} e^{-\lambda_{\min } \nu_{d}|y|^{d} / 2^{d+1}}\left(1-e^{-2 \beta d \nu_{d} \mathbf{E}\left[\rho^{d-1}\right]|y|}\right) \mathrm{d} y \\
& =\frac{2^{d^{2}} \nu_{d}}{\lambda_{(\Xi)}} \frac{\lambda_{\max }^{d}}{\lambda_{\min }^{d-2}} \int_{0}^{\infty} t^{d-1} e^{-\lambda_{\min } \nu_{d} t^{d} / 2^{d+1}}\left(1-e^{-2 \beta d \nu_{d} \mathbf{E}\left[\rho^{d-1}\right] t}\right) \mathrm{d} t .
\end{aligned}
$$

Note that the remainder $R$ tends to 0 when $\beta \rightarrow 0$ and $\mathbf{E}\left[\rho^{d}\right] \rightarrow \infty$ such that $\beta \mathbf{E}\left[\rho^{d}\right]=$ const; that is, when the grains become large and sparsely distributed while the volume fraction of the $\mathrm{BM} \mathbb{X}$ is constant. Figure 2 shows the bound (5.6) for the 2-dimensional model $(d=2)$ with a deterministic radius $\rho$. The curves correspond to different (constant) volume fractions of the Boolean Model with varying radius $\rho$ of its individual ball. From top to bottom:

- the volume fraction of the Boolean model $p_{1} \equiv 1-e^{-\beta \pi \rho^{2}}=0.86$ with $\lambda_{1}=5, \lambda_{2}=10$ and $\lambda_{1}=10, \lambda_{2}=5$,

- $p_{1}=0.63$ with $\lambda_{1}=5, \lambda_{2}=10$ and $\lambda_{1}=10, \lambda_{2}=5$,

- $p_{1}=0.39$ with $\lambda_{1}=10, \lambda_{2}=5$ and $\lambda_{1}=5, \lambda_{2}=10$,

- $p_{1}=0.31$ with $\lambda_{1}=10, \lambda_{2}=5$ and $\lambda_{1}=5, \lambda_{2}=10$. 


\section{Acknowledgments}

The authors wish to thank F. Baccelli for proposing the problem faced in this work. This research has been partly supported by RNRT project GEORGES and KBN grant 2 P03A 02023.

\section{References}

[1] Baccelli, F., BŁaszczyszyn, B. And Tournois, F. (2003). Downlink capacity and admission/congestion control in CDMA networks. In submitted to IEEE INFOCOM'03. New-York.

[2] Baccelli, F., Klein, M., Lebourges, M. And Zuyev, S. (1997). Stochastic geometry and architecture of communication networks. Telecommunications systems $\mathbf{7}, 209-227$.

[3] Baccelli, F. And Zuyev, S. (1997). Stochastic geometry models of mobile communication networks. In Frontiers in queueing. Models and Applications in Science and Engineering. ed. J. Dshalalow. CRC Press, Boca Raton pp. 227-244.

[4] Calka, P. (2002). The law of the smallest disk containing the typical Poisson-Voronoi cell. C. R. Math. Acad. Sci. Paris 334, 325-330.

[5] Courtois, P. (1977). Decomposability, Queueing and Computer System Applications. Academic Press, New York, New York.

[6] Goldman, A. And Calka, P. (2001). On the spectral function of Poisson-Voronoi cells. C. R. Math. Acad. Sci. Paris 332, 835-840.

[7] Gupta, P. And Kumar, P. R. (2000). The capacity of wireless networks. IEEE Transactions on Information Theory 46, 388-404.

[8] Hayen, A. And Quine, M. (2000). The proportion of triangles in a Poisson-Voronoi tessellation of the plane. Adv. in Appl. Probab. 32, 67-74.

[9] Hayen, A. And Quine, M. (2002). Areas of components of a Voronoi polygon in a homogeneous Poisson process in the plane. Adv. in Appl. Probab. 34, 281-291.

[10] Matheron, G. (1975). Random Sets and Integral Geometry. John Willey \& Sons, London.

[11] MøLler, J. (1989). Lectures on Random Voronoi Tessellations vol. 87 of Lecture Notes in Statistics. Springer-Verlag, New York.

[12] Okabe, A., Boots, B. And Sugihara, K. (1995). Spatial Tessellations. John Willey \& Sons, Chichester.

[13] Stoyan, D., Kendall, W. And Mecke, J. (1995). Stochastic Geometry and its Applications. Wiley, Chichester. 
Unité de recherche INRIA Rocquencourt Domaine de Voluceau - Rocquencourt - BP 105 - 78153 Le Chesnay Cedex (France)

Unité de recherche INRIA Lorraine : LORIA, Technopôle de Nancy-Brabois - Campus scientifique 615, rue du Jardin Botanique - BP 101 - 54602 Villers-lès-Nancy Cedex (France)

Unité de recherche INRIA Rennes : IRISA, Campus universitaire de Beaulieu - 35042 Rennes Cedex (France)

Unité de recherche INRIA Rhône-Alpes : 655, avenue de l'Europe - 38330 Montbonnot-St-Martin (France)

Unité de recherche INRIA Sophia Antipolis : 2004, route des Lucioles - BP 93 - 06902 Sophia Antipolis Cedex (France) 